\title{
A comprehensive dosimetric study on switching from a Type-B to a Type-C dose algorithm for modern lung SBRT
}

Christina Zhou', Nathan Bennion², Rongtao Ma², Xiaoying Liang ${ }^{3}$, Shuo Wang ${ }^{2}$, Kristina Zvolanek ${ }^{4}$, Megan Hyun², Xiaobo Li ${ }^{2,5}$, Sumin Zhou ${ }^{2}$, Weining Zhen ${ }^{2}$, Chi Lin ${ }^{2}$, Andrew Wahl ${ }^{2}$ and Dandan Zheng ${ }^{2 *}$

\begin{abstract}
Background: Type-C dose algorithms provide more accurate dosimetry for lung SBRT treatment planning. However, because current dosimetric protocols were developed based on conventional algorithms, its applicability for the new generation algorithms needs to be determined. Previous studies on this issue used small sample sizes and reached discordant conclusions. Our study assessed dose calculation of a Type- $C$ algorithm with current dosimetric protocols in a large patient cohort, in order to demonstrate the dosimetric impacts and necessary treatment planning steps of switching from a Type-B to a Type-C dose algorithm for lung SBRT planning.

Methods: Fifty-two lung SBRT patients were included, each planned using coplanar VMAT arcs, normalized to $D_{95 \%}=$ prescription dose using a Type-B algorithm. These were compared against three Type-C plans: re-calculated plans (identical plan parameters), re-normalized plans ( $\mathrm{D}_{95 \%}=$ prescription dose), and re-optimized plans. Dosimetric endpoints were extracted and compared among the four plans, including RTOG dosimetric criteria: $\left(R_{100 \%}, R_{50 \%}\right.$ $\mathrm{D}_{2 \mathrm{~cm}}, \mathrm{~V}_{105 \%}$, and lung $\left.\mathrm{V}_{20}\right)$, PTV $\mathrm{D}_{\min }, \mathrm{D}_{\max }, \mathrm{D}_{\text {mean, }} \mathrm{V}_{\%}$ and $\mathrm{D}_{90 \%}$, PTV coverage $\left(\mathrm{V}_{100 \%}\right)$, homogeneity index $(\mathrm{HI})$, and Paddick conformity index (PCl).

Results: Re-calculated Type-C plans resulted in decreased PTV D $D_{\min }$ with a mean difference of $5.2 \%$ and increased $D_{\max }$ with a mean difference of 3.1\%, similar or improved RTOG dose compliance, but compromised PTV coverage (mean $\mathrm{D}_{95 \%}$ and $\mathrm{V}_{100 \%}$ reduction of 2.5 and $8.1 \%$, respectively). Seven plans had $>5 \% \mathrm{D}_{95 \%}$ reduction (maximum reduction $=16.7 \%$ ), and 18 plans had $>5 \% \bigvee_{100 \%}$ reduction (maximum reduction $=60.0 \%$ ). Re-normalized Type- $C$ plans restored target coverage, but yielded degraded plan conformity (average PCI reduction 4.0\%), and RTOG dosimetric criteria deviation worsened in 11 plans, in $R_{50 \%}, D_{2 c m}$ and $R_{100 \%}$. Except for one case, re-optimized Type-C plans restored RTOG compliance achieved by the original Type-B plans, resulting in similar dosimetric values but slightly higher target dose heterogeneity (mean $\mathrm{HI}$ increase $=13.2 \%$ ).
\end{abstract}

Conclusions: Type-B SBRT lung plans considerably overestimate target coverage for some patients, necessitating Type-C re-normalization or re-optimization. Current RTOG dosimetric criteria appear to remain appropriate.

Keywords: Lung, SBRT, Dose algorithms, Monte Carlo, VMAT

\footnotetext{
* Correspondence: sabrinadan@gmail.com

${ }^{2}$ Department of Radiation Oncology, University of Nebraska Medical Center,

42nd and Emile St, Omaha, NE 68198, USA

Full list of author information is available at the end of the article
} 


\section{Background}

Lung cancer is the second most common type of cancer for both men and women, and the leading cause of cancer death, making up about 1 in every 4 cancer-related deaths [1]. Non-small cell lung cancer (NSCLC) makes up about $80-85 \%$ of all cases. Treatment for NSCLC varies depending on the cancer stage and other patient factors, but some common treatments include surgery, chemotherapy, immunotherapy, and radiation therapy. Stereotactic body radiotherapy (SBRT), also known as stereotactic ablative radiotherapy (SABR), is a type of external beam radiotherapy that is often used to treat early stage lung cancers as an increasingly popular alternative option to surgery [2-4]. It is also often used to treat small-sized, oligo-metastasis in the lung [5].

Lung SBRT conventionally used multiple coplanar or non-coplanar conformal beams [6]. In recent years, volumetric-modulated arc therapy (VMAT) has gained greater popularity as the treatment technique of choice for lung SBRT because of the quick delivery time, superior critical tissue sparing and dose conformity, and robustness against the interplay effect due to respiratory motion [7-12]. Several studies showed a substantial reduction in the treatment time for SBRT lung cases using VMAT when compared to intensity-modulated radiation therapy (IMRT) and conformal beam treatment, from dozens of to just a few minutes, especially when combined with the high dose rates provided by flattening-filter-free modes of modern linear accelerators (LINACs) [7, 9, 11]. While offering comparable steep dose gradients and critical tissue sparing to IMRT, VMAT was also found to be less susceptible to the interplay effect that prevented the wide application of IMRT in thoracic radiotherapy, especially when conventional dose rates are used [8, 10, 12]. However, accurate dose calculation in the presence of heterogeneity remains a challenge that arises when using SBRT [13-18]. The heterogeneous tissue interfaces between low density lung tissue and high density tumor pose challenges for accurate dose modeling. Starting from homogeneous dose calculation, commercial treatment planning systems progressed in generations of heterogeneity corrected calculation algorithms, often classified as Type-A to Type- $\mathrm{C}$, to better address this challenge [19-21]. Conventional homogeneous dose calculations and Type-A or pencil beam algorithms with equivalent path length corrections can often lead to target peripheral dose over-estimation as high as 50\% [22-25]. As the current mainstay of commercial treatment planning algorithms, Type-B or convolution/superposition dose algorithms are commonly used due to their improved accuracy compared with older algorithms [17, 26]. On the other hand, studies have shown that while these algorithms resulted in $<3 \%$ errors on the peripheral target dose in many patient cases, errors could be as high as over
10\% compared with Monte Carlo calculation in other cases [27-30]. Therefore, Type- $\mathrm{C}$ or fast Monte Carlo algorithms have been implemented in commercial treatment planning systems in the recent decade, which provide improved dose calculation accuracy over Type-B algorithms but faster dose modeling than full-fledged Monte Carlo simulation [29, 31-33]. Acuros XB dose calculation algorithm, implemented for treatment planning in Eclipse (Varian Medical Systems, Palo Alto, CA), is based on a linear Boltzmann equation solver to solve radiation transport equation, rather than simulation of particle transport implemented in Monte Carlo. It is considered a Type- $C$ algorithm due to the comparable level of dose modeling accuracy [30, 34-36].

Many studies have investigated the dosimetric differences between Type-A or Type-B and Type-C dose algorithms, and have shown that Type- $\mathrm{C}$ algorithms more accurately calculate the dose distribution for SBRT plans of lung patients [15, 22-24, 27, 32, 35]. Due to the large dose errors associated with Type-A algorithms, they have been gradually phased out in thoracic treatment planning. Modern lung SBRT protocols from National Research Group (NRG)/Radiation Therapy Oncology Group (RTOG) such as RTOG0813 and RTOG0915 require Type-B dose calculation [37, 38]. Despite the demonstrated dose calculation accuracy improvement and the increasing availability, currently the clinical utilization of Type- $\mathrm{C}$ algorithms is still limited [5]. In addition to technical challenges such as lengthier computation time and sub-optimal or lack of integration into inverse optimization engines compared with Type-B algorithms, there are also some clinical challenges. Firstly, inhomogeneous dose errors were revealed for Type-B compared with Type-C algorithms for different patients. While large errors can result for some patients, small errors within 3\% were found for other patients [27-30], suggesting that elaborate dose calculation using Type-C algorithms may not be necessary for every lung SBRT patient to offset the lengthier computation time. Secondly, current clinical guidelines on dose prescription and constraints have been established for Type-B algorithms and may not be directly applicable for Type-C algorithms.

Several authors have previously examined the dosimetric compliance of Type-C calculated lung SBRT plans to the current protocols $[27,29,39,40]$. However, discordant recommendations were reported by these studies. Li et al. applied Monaco (Elekta/CMS, Crawley, UK), a Type-C algorithm, on 15 patients planned using $\mathrm{XiO}$ (Elekta/CMS, Crawley, UK), a Type-B algorithm, to examine RTOG 0813 compliance [29]. They recommended adjusting the dosimetric criteria (such as $\mathrm{R}_{50 \%}$ ) because their values were increased with the Type- $\mathrm{C}$ algorithm and the corresponding compliance was worse. In contrast, Rana et al. published a study on 14 patients evaluating the effect of 
switching from a Type-B algorithm (Analytical Anisotropic Algorithm, or AAA) to a Type-C algorithm (AXB) in Eclipse for lung SBRT plans using RTOG0813 dosimetric criteria [27]. They found that the AXB recalculation of the original AAA plans led to lower average values for most dose constraint parameters and reduced instances of protocol minor deviations on these parameters. Two studies by Pokhrel et al. used another Type-C algorithm, Voxel Monte Carlo implemented in iPlan (BrainLab AG, Feldkirchen, Germany), to evaluate the plan compliance with dosimetric criteria of RTOG0813 and RTOG0915 for central and peripheral lung SBRT patients, respectively $[39,40]$. Based on the average values obtained on 20 patients, Pokhrel et al. recommended the necessary adjustments to the dosimetric criteria in order to make their Type-C plans fully compliant with the protocols.

The aim of this study, therefore, was to systematically investigate the necessity and dosimetric impacts of switching from Type- $\mathrm{B}$ to Type-C dose algorithms for lung SBRT planning on a large patient cohort. On fifty-two patients, the target dose parameters as well as RTOG dosimetric criteria were compared among the following plans: original Type-B plans, re-calculated Type-C plans with identical beam settings, re-normalized Type-C plans (to ensure target coverage), and re-optimized Type-C plans (to restore the original compliance to the dosimetric criteria achieved by the Type-B plans).

\section{Methods}

As will be detailed in the following sections, the dosimetric impacts of switching from Type- $\mathrm{B}$ to Type-C calculation were comprehensively evaluated using various dosevolume endpoints and protocol dosimetric constraints on 52 lung SBRT patient plans. We compared the original Type-B plans with the re-calculated, re-normalized, and re-optimized Type-C plans.

\section{Original Type-B plans}

With the approval of the University of Nebraska Medical Center institutional review board, treatment data for 70 patients with NSCLC, treated with lung SBRT at our institution between April 2014 and August 2016, were collected. From these, 52 plans treated with VMAT were selected for this retrospective study. The remaining cases were excluded because they used 3D conformal treatment techniques.

According to our institutional protocol, each patient received a free-breathing three-dimensional computed tomography (3D CT) followed by an eight-phase fourdimensional computed tomography (4D CT) acquired using a Sensation Open CT simulator (Siemens, Erlangen, Germany) and the Real-time Position Management system (Varian Medical Systems, Palo Alto, CA) as the respiratory surrogate. A slice thickness of $2 \mathrm{~mm}$ was used for both CT scans. Patients were simulated in treatment position and immobilized with the BlueBAG ${ }^{\text {ma }}$ immobilization system (Medical Intelligence, Schwabmünchen, Germany). The 3D and 4D CT images were fused using the common frame of reference if visually confirmed with no relative movement, or otherwise using rigid registration to the spine. For each patient, the gross tumor volume (GTV) was delineated by the attending radiation oncologists as the visible tumor on the $3 \mathrm{D} \mathrm{CT}$, and the internal target volume (ITV) was contoured using the 3DCT, maximum intensity projection (MIP) from the 4DCT, and each phase of the 4DCT, as the union of the gross tumor seen on these images excluding possible nearby soft-tissue. The planning target volume (PTV) was generated by adding an isotropic expansion of 5-6 $\mathrm{mm}$ to the ITV.

For treatment planning, one to two coplanar VMAT arcs were used, with partial arcs on the ipsilateral side to spare the contralateral lung for some peripheral lesions and full arcs for the rest. Fifty plans used flatteningfilter-free $6 \mathrm{MV}$ and/or $10 \mathrm{MV}$ beams, and the rest used flattened beams of these energies. The plans were optimized to best comply with the dosimetric criteria and normal tissue constraints specified in RTOG0813, RTOG0915, other guidelines [37, 38, 41], and according to our institutional protocol. On average, the prescription dose was about $88 \%$ of the maximum dose, with the lowest prescription isodose line at $76 \%$ of the maximum dose for one plan. All the treatment plans specified a dose prescription of either 48 Gy (12 Gy x 4 fractions) or 50 Gy (10 Gy x 5 fractions). Additionally, all cases were planned using Type-B algorithms, with 47 plans using Analytical Anisotropic Algorithm, or AAA in Eclipse 13.5 (Varian Medical Systems, Palo Alto, CA) and 5 plans using collapsed cone convolution, or CCC in Pinnacle [3] (Philips Medical, Fitchburg WI). Plans originally planned in Pinnacle [3] were transferred into Eclipse and re-calculated with AAA for this study. All plans were normalized so that $95 \%$ of the PTV received the full prescription dose $\left(\mathrm{D}_{95 \%}=\right.$ prescription dose $)$.

\section{Re-calculated Type-C plans}

The above plans were re-calculated using a Type- $C$ algorithm, Acuros XB or AXB in Eclipse 13.5. Identical beam parameters including MLC patterns and monitor units were used for the re-calculation as in the original Type-B, or AAA plans. For AXB calculations, dose-tomedium was reported.

\section{Re-normalized Type-C plans}

Another set of treatment plans was generated by recalculating the original clinical treatment plans with the Type-C algorithm (AXB), but specifying plan normalization so that $95 \%$ of the target volume received 
the full prescription dose $\left(D_{95 \%}=\right.$ prescription dose $)$ as in the original clinical Type- $\mathrm{B}$ plans. In other words, these plans used identical MLC patterns and relative beam weighting as the original plans, but with different MUs so that the Type-C plans' PTV doses were re-normalized to match the original Type-B, or AAA plans $\left(D_{95 \%}=\right.$ prescription dose).

\section{Re-optimized Type-C plans}

For the re-normalized Type-C plans that did not meet the level of RTOG dosimetric compliance seen in their respective original Type-B plans, re-optimization was performed using the Type-C algorithm, keeping the normalization at $D_{95 \%}=$ prescription dose, to improve the dosimetric compliance. Specifically, in addition to dose objectives for critical organs, the following dosimetric criteria from the protocols were utilized: ratio of prescription isodose volumes to PTV $\left(\mathrm{R}_{100 \%}\right)$, ratio of $50 \%$ prescription isodose volume to PTV $\left(\mathrm{R}_{50 \%}\right)$, maximal dose $2 \mathrm{~cm}$ from the PTV in any direction as a percentage of prescription dose $\left(D_{2 \mathrm{~cm}}\right)$, the percentage of lung receiving dose equal to or larger than $20 \mathrm{~Gy}\left(\mathrm{~V}_{20}\right)$, and the volume of $105 \%$ isodose outside the PTV $\left(\mathrm{V}_{105 \%}\right)$. The optimization objectives were adjusted to create plans that optimize these dosimetric values with intermediate and final Type-C dose calculation.

\section{Dosimetric evaluation and comparison}

For all above plans, the minimum $\left(D_{\min }\right)$, maximum $\left(D_{\text {max }}\right)$, and mean $\left(D_{\text {mean }}\right)$ doses of the PTV were recorded, along with the doses received by $95 \%\left(D_{95 \%}\right)$ and 90\% $\left(D_{90 \%}\right)$ of the PTV volume. Volume-based dosimetric parameters such as $D_{95 \%}$ were included and used for plan normalization because single-point-based doses such as $\mathrm{D}_{\min }$ are associated with higher uncertainties. The PTV coverage, referring to the percent volume of PTV covered by prescription dose or $\mathrm{V}_{100 \%}$, was also recorded. RTOG criteria were used to calculate the values for $R_{100 \%}, R_{50 \%}$, $\mathrm{D}_{2 \mathrm{~cm}}, \mathrm{~V}_{20}$, and $\mathrm{V}_{105 \%}$. Additionally, the Paddick Conformity Index (PCI) [42] and the homogeneity index (HI) [43] were calculated. The PCI was defined as $\left(\mathrm{TV}_{\mathrm{PIV}}\right)^{2} /(\mathrm{TV} \mathrm{x}$ PIV), with $\mathrm{TV}_{\mathrm{PIV}}$ being the PTV volume receiving full prescription dose, TV being the total PTV volume, and PIV being the total irradiated volume receiving full prescription dose. For any plan, the PCI would be within the range of 0 to 1.0 , with $\mathrm{PCI}=1.0$ for an ideal plan. The HI was defined as the ratio of the maximum dose over the prescription dose. The above dosimetric parameters were used to compare the four types of plans as described in II.A.-II.D. for each of the 52 patients.

\section{Results}

\section{Clinical characteristics}

Patient and tumor characteristics of the 52 patients are described in Table 1.
Table 1 Patient and tumor characteristics

\begin{tabular}{ll}
\hline Parameter & Total \\
\hline Patients $(n=52)$ & Female $=26$, male $=26$ \\
Median age in years (range) & $73(46-89)$ \\
Median PTV in $\mathrm{cm}^{3}$ (range) & $22.4(6.1-85.7)$ \\
Tumor location $(n=52)$ & $13 \mathrm{LUL}, 14 \mathrm{RUL}, 7 \mathrm{LLL}, 14 \mathrm{RLL}, 4 \mathrm{RML}$ \\
\hline
\end{tabular}

LUL left upper lobe; RUL right upper lobe; LLL left lower lobe; RLL right lower lobe; $R M L$ right middle lobe

\section{Re-calculated and re-normalized Type-C plans vs. original Type-B plans}

For the 52 patients, the average values and standard deviations are summarized in Table 2 for PTV dosimetric parameters, and in Table 3 for PTV coverage $\left(\mathrm{V}_{100 \%}\right)$, $\mathrm{PCI}$, and $\mathrm{HI}$. The numbers of cases with deviations to RTOG dosimetric criteria are listed in Table 4. These were used to compare between re-calculated Type-C plans, re-normalized Type-C plans, and the original Type-B plans.

As shown in Table 4, re-calculated Type-C plans resulted in comparable or improved compliance for RTOG dose criteria compared with the original Type-B plans. Of the original 52 Type-B plans, 30 plans had minor deviations for $R_{50 \%}$ and 16 had minor deviations for $D_{2 \mathrm{~cm}}$. None had deviations for $R_{100 \%}, V_{20}$, or $V_{105 \%}$, and no major deviations were observed for any of the criteria. After re-calculating with Type-C algorithm, the level of deviation was reduced from minor to none for 3 plans for $\mathrm{R}_{50 \%}$ and similarly in 5 plans for $\mathrm{D}_{2 \mathrm{~cm}}$. On the other hand, these re-calculated Type- $C$ plans revealed the target coverage dose overestimation by the Type- $\mathrm{B}$ algorithm, as can be seen in the reduced PTV $\mathrm{D}_{\min }$, $\mathrm{D}_{95 \%}$, and $\mathrm{D}_{90 \%}$ in Table 2, and in the reduced PTV coverage or $\mathrm{V}_{100 \%}$ in Table 3. It is worth noting that although the $2.3 \%$ average PTV $\mathrm{D}_{95 \%}$ reduction may not seem clinically significant, a wide range of variation (standard deviation $=4.4 \%$ ) was observed from patient to patient, with a maximum reduction of $16.7 \%$. Of the 52 patients, 7 patients $(13.5 \%)$ showed PTV $\mathrm{D}_{95 \%}$ a reduction over $5 \%$, and 4 patients $(7.7 \%)$ showed a reduction over $10 \%$. Figure 1 plots the distribution of the magnitude for PTV $D_{95 \%}$ reduction. Similarly, PTV

Table 2 PTV dosimetric data averages and standard deviations over all patients for the original Type-B plans and the re-calculated as well as re-normalized Type-C comparison plans

$D_{\min } \quad D_{\text {mean }} \quad D_{\max } \quad D_{95 \%} \quad D_{90 \%}$

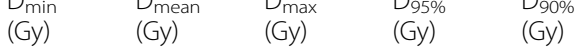

\begin{tabular}{llllll}
\hline Original Type-B & $45.5 \pm 2.6$ & $52.5 \pm 1.6$ & $56.5 \pm 3.8$ & $49.5 \pm 0.9$ & $50.1 \pm 1.0$
\end{tabular} Re-calculated $\quad 43.2 \pm 3.4 \quad 52.5 \pm 2.3 \quad 59.6 \pm 3.4 \quad 48.4 \pm 2.9 \quad 49.3 \pm 2.4$ Type-C

Re-normalized $\quad 44.1 \pm 2.4 \quad 53.7 \pm 1.9 \quad 59.6 \pm 3.9 \quad 49.5 \pm 0.9 \quad 50.5 \pm 0.9$ Type-C 
Table 3 PTV coverage (defined as $V_{100 \%}$ ), Paddick conformity index $(\mathrm{PCl})$, and homogeneity index $(\mathrm{HI})$ averages and standard deviations over all patients for the original Type-B plans and recalculated as well as re-normalized Type-C comparison plans

\begin{tabular}{lcll}
\hline & PTV Coverage $\left(\mathrm{V}_{100 \%}\right)(\%)$ & $\mathrm{PCl}$ & $\mathrm{HI}$ \\
\hline Original Type- $\mathrm{B}$ & $92.2 \pm 3.3$ & $0.9 \pm 0.1$ & $1.1 \pm 0.1$ \\
Re-calculated Type-C & $84.8 \pm 14.6$ & $0.8 \pm 0.1$ & $1.2 \pm 0.1$ \\
Re-normalized Type-C & $92.7 \pm 2.5$ & $0.8 \pm 0.1$ & $1.2 \pm 0.1$ \\
\hline
\end{tabular}

$\mathrm{V}_{100 \%}$ was reduced on average by $7.4 \%$ from $92.2 \pm 3.3 \%$ to $84.8 \pm 14.6 \%$. Of the 52 patients, 18 patients $(34.6 \%)$ showed a PTV $\mathrm{V}_{100 \%}$ reduction over $5 \%$, and 15 patients $(28.8 \%)$ showed a reduction over $10 \%$. The maximum $\mathrm{V}_{100 \%}$ reduction was $60 \%$ for one patient. Figure 2 plots the PTV $\mathrm{D}_{95 \%}$ and $\mathrm{V}_{100 \%}$ reductions for all patients over PTV size.

The PTV coverage reductions on the re-calculated Type-C plans suggested that simply re-calculating the plans with the Type-C algorithm might not provide fair comparisons. For example, on the 8 plans with improved RTOG dose compliance on the re-calculated Type-C plans when compared with the original Type-B plans, the average PTV $\mathrm{D}_{95 \%}$ reduction was $4.2 \%$, with a maximum reduction of $15.1 \%$. We therefore designed renormalized Type-C plans to restore the dose coverage by ensuring $\mathrm{D}_{95 \%}=$ prescription dose through inflated MUs. Not surprisingly, the average values for the RTOG dosimetric criteria increased on the re-normalized plans for $R_{100 \%}, R_{50 \%}$, and $D_{2 \mathrm{~cm}}$ compared with the original Type-B plans. On 11 out of the 52 plans (21.2\%), protocol compliance degraded on one or more dosimetric criteria when compared with the original Type-B plans. For $\mathrm{R}_{50 \%}, 5$ plans changed from minor to major deviation, and 4 plans from no deviation to minor deviation. For $\mathrm{D}_{2 \mathrm{~cm}}, 1$ plan changed from minor to major deviation, 6 plans from no deviation to minor deviation, and 5 plans from minor deviation to no deviation. For $\mathrm{R}_{100 \%}, 3$ plans changed from no deviation to minor deviation. No plans showed changes for $\mathrm{V}_{105 \%}$ or lung $\mathrm{V}_{20}$.

The ratios of the re-calculated and re-normalized Type-C plans over the original Type-B plans are plotted against PTV volume in Fig. 3(a)-(d) for RTOG dosimetric parameters $\mathrm{R}_{100 \%}, \mathrm{R}_{50 \%}, \mathrm{D}_{2 \mathrm{~cm}}$, and lung

Table 4 RTOG criteria compliance (numbers of cases with deviations) for the original Type-B plans and re-calculated as well as re-normalized Type-C comparison plans

\begin{tabular}{llll}
\hline & $\mathrm{R}_{100 \%}$ & $\mathrm{R}_{50 \%}$ & $\mathrm{D}_{2 \mathrm{~cm}}$ \\
\hline Original Type-B & 0 deviation & 30 minor deviations & 16 minor deviations \\
Re-calculated & 0 deviation & 27 minor deviations & 11 minor deviations \\
Type-C & & & \\
Re-normalized & 3 minor & 29 minor deviations, & 16 minor deviations, \\
Type-C & deviations & 5 major deviations & 1 major deviation \\
\hline
\end{tabular}

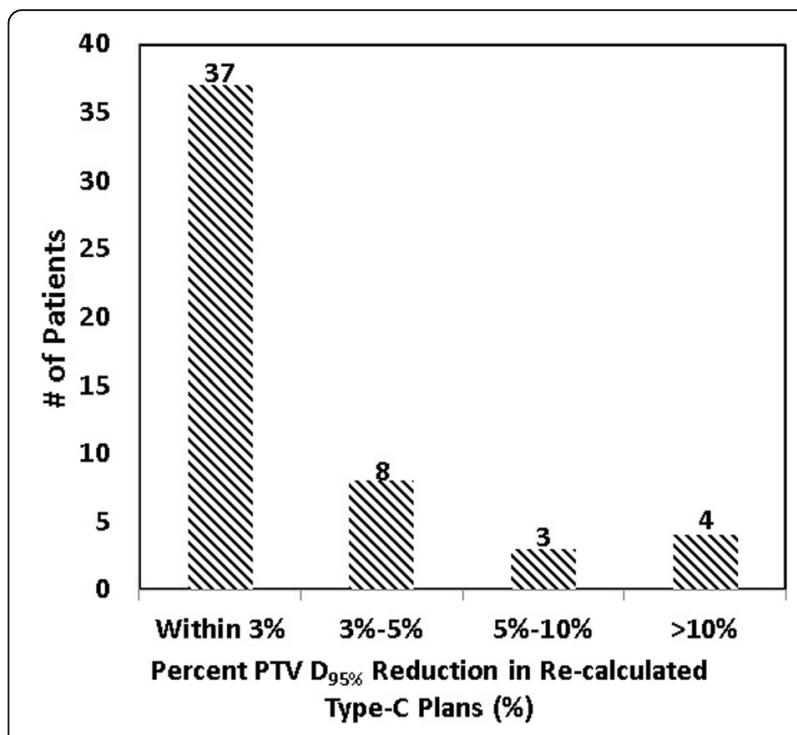

Fig. 1 Distribution of the magnitude for PTV $D_{95 \%}$ reduction on re-calculated Type-C plans

$\mathrm{V}_{20}$, respectively. It is apparent that the ratios for the re-calculated plans are more clustered around or below 1.0 and those for the re-normalized plans are more spread out and above 1.0. This indicates that while re-calculated Type-C plans achieved similar or even better RTOG dosimetric criteria compliance as the original Type- $\mathrm{B}$ plans, the compliance is worsened on the re-normalized Type-C plans in which adequate target dose coverage was restored.

\section{Re-optimized Type-C plans}

For the 11 patients whose re-normalized Type-C plans showed worsened RTOG dosimetric criteria compliance for $R_{50 \%}, D_{2 \mathrm{~cm}}$ and/or $R_{100 \%}$, re-optimization was performed using the Type-C algorithm. While keeping the original beam arrangements and collimator angles,

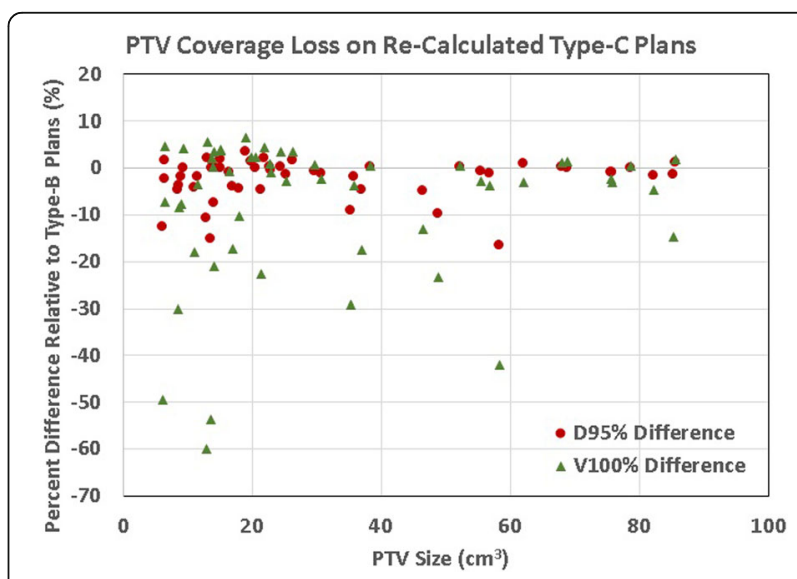

Fig. 2 PTV coverage loss (as in $D_{95 \%}$ and $V_{100 \%}$ reductions) on re-calculated Type-C plans over PTV size 

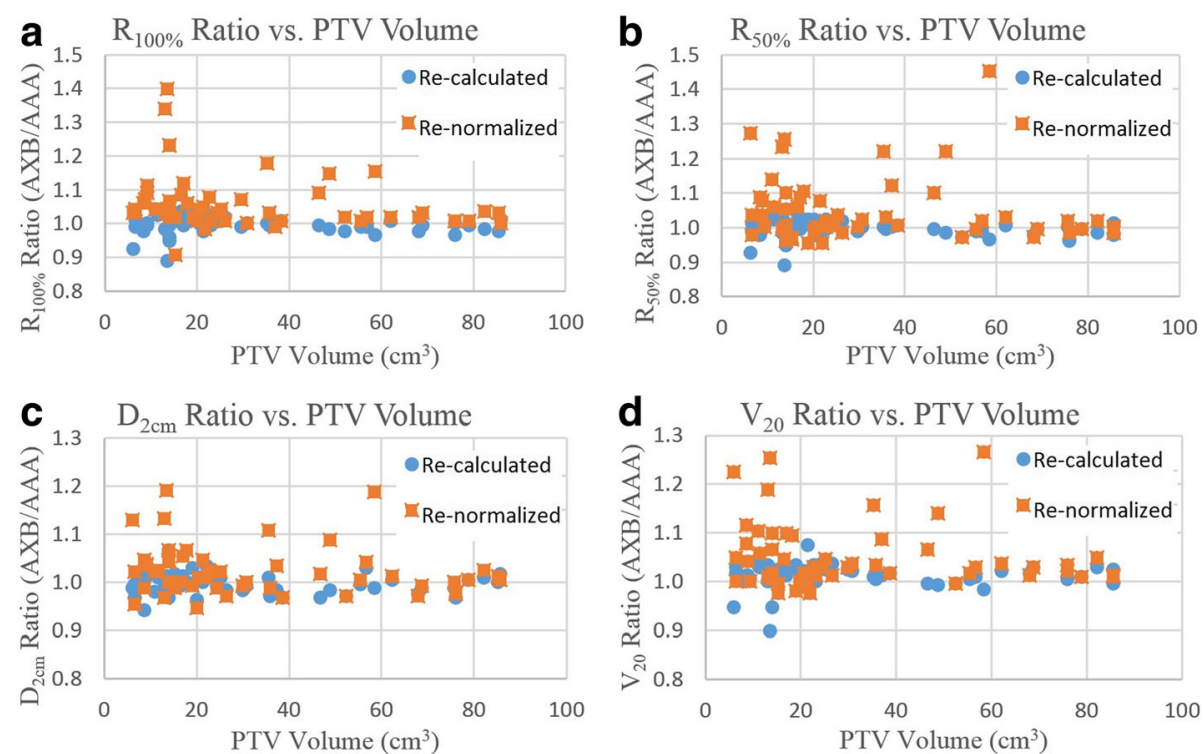

Fig. 3 The ratios of the re-calculated and re-normalized Type-C plans over the original Type-B plans are plotted against PTV volume for RTOG dosimetric parameters (a) $R_{100 \%}$ (b) $R_{50 \%}$ (c) $D_{2 \mathrm{~cm}}$, and (d) lung $V_{20}$

new optimization objectives were necessary for Type-C optimization, different from those used for the original Type-B optimization. On these 11 patients, the ratios of the re-optimized Type-C plan over the original Type-B plan were listed for PTV dosimetric parameters, RTOG criteria, and other plan quality indexes in Tables 5, 6, and 7 , respectively. The PTV volumes and the PTV $\mathrm{D}_{95 \%}$ reduction revealed by the re-calculated plans are also listed alongside as a reference. The original levels of compliance on the original Type-B plans were restored by the re-optimization for all but one patient, Patient

Table 5 PTV dosimetric parameter ratios of the re-optimized Type-C plan over the original Type-B plan for the 11 re-optimized patients

\begin{tabular}{|c|c|c|c|c|c|c|c|}
\hline Pt \# & $\begin{array}{l}\text { PTV Volume } \\
\left(\mathrm{cm}^{3}\right)\end{array}$ & $\begin{array}{l}D_{95 \%} \text { Reduction } \\
(\%)\end{array}$ & $\begin{array}{l}\text { Ratio } \\
D_{\min }\end{array}$ & $\begin{array}{l}\text { Ratio } \\
D_{\text {mean }} \\
\end{array}$ & $\begin{array}{l}\text { Ratio } \\
D_{\max } \\
\end{array}$ & $\begin{array}{l}\text { Ratio } \\
D_{95 \%} \\
\end{array}$ & $\begin{array}{l}\text { Ratio } \\
\mathrm{D}_{90 \%} \\
\end{array}$ \\
\hline 1 & 6.1 & 12.5 & 0.9 & 1.1 & 1.2 & 1.0 & 1.0 \\
\hline 2 & 6.5 & 2.3 & 0.9 & 1.1 & 1.2 & 1.0 & 1.0 \\
\hline 3 & 13.0 & 10.6 & 1.0 & 1.1 & 1.1 & 1.0 & 1.0 \\
\hline 4 & 13.6 & 15.1 & 0.9 & 1.1 & 1.1 & 1.0 & 1.0 \\
\hline 5 & 14.1 & 7.4 & 1.0 & 1.0 & 1.1 & 1.0 & 1.0 \\
\hline 6 & 17.0 & 4.1 & 1.0 & 1.1 & 1.2 & 1.0 & 1.0 \\
\hline 7 & 18.0 & 4.5 & 1.0 & 1.0 & 1.0 & 1.0 & 1.0 \\
\hline 8 & 35.4 & 9.1 & 1.0 & 1.0 & 1.0 & 1.0 & 1.0 \\
\hline 9 & 37.1 & 4.7 & 1.0 & 1.0 & 1.0 & 1.0 & 1.0 \\
\hline 10 & 48.8 & 9.7 & 0.9 & 1.2 & 1.4 & 1.0 & 1.0 \\
\hline 11 & 58.4 & 16.7 & 1.0 & 1.1 & 1.1 & 1.0 & 1.0 \\
\hline
\end{tabular}

Also listed are the PTV volumes and PTV $D_{95 \%}$ reduction revealed by the corresponding re-calculated Type- $C$ plans as a reference. The patients are sorted by the PTV size
\#11, who continued to have a minor $R_{50 \%}$ deviation after re-optimization while the original Type-B plan had no deviation.

As an example of comparing the 4 plans of the same patient, the isodose distributions in the axial view at the isocenter for Patient \#3 (in Tables 5, 6, and 7) are placed side by side in Fig. 4. Compared with the original TypeB plan (a), the PTV underdose on the re-calculated Type-C plan (b) is apparent. While the re-normalized Type-C plan (c) restored the target dose coverage, the

Table 6 RTOG dosimetric parameter ratios of the re-optimized Type-C plan over the original Type-B plan for the 11 re-optimized patients

\begin{tabular}{lcccccc}
\hline Pt \# & $\begin{array}{l}\text { PTV Volume } \\
\left(\mathrm{cm}^{3}\right)\end{array}$ & $\begin{array}{l}D_{95 \%} \text { Reduction } \\
(\%)\end{array}$ & $\begin{array}{l}\text { Ratio } \\
\mathrm{R}_{100 \%}\end{array}$ & $\begin{array}{c}\text { Ratio } \\
\mathrm{R}_{50 \%}\end{array}$ & $\begin{array}{c}\text { Ratio } \\
\mathrm{D}_{2 \mathrm{~cm}}\end{array}$ & $\begin{array}{l}\text { Ratio } \\
V_{20}\end{array}$ \\
\hline 1 & 6.1 & 12.5 & 1.0 & 1.2 & 1.1 & 1.2 \\
2 & 6.5 & 2.3 & 1.0 & 0.8 & 1.0 & 0.8 \\
3 & 13.0 & 10.6 & 0.9 & 0.9 & 1.0 & 1.0 \\
4 & 13.6 & 15.1 & 1.0 & 0.9 & 1.0 & 1.0 \\
5 & 14.1 & 7.4 & 0.9 & 0.8 & 1.0 & 0.9 \\
6 & 17.0 & 4.1 & 1.0 & 0.8 & 1.0 & 0.9 \\
7 & 18.0 & 4.5 & 1.0 & 1.1 & 1.0 & 1.2 \\
8 & 35.4 & 9.1 & 1.0 & 1.1 & 0.9 & 1.2 \\
9 & 37.1 & 4.7 & 0.9 & 1.0 & 1.0 & 1.0 \\
10 & 48.8 & 9.7 & 1.0 & 1.0 & 1.0 & 1.0 \\
11 & 58.4 & 16.7 & 1.0 & $1.2^{\mathrm{a}}$ & 1.0 & 1.2 \\
\hline
\end{tabular}

Also listed are the PTV volumes and PTV $D_{95 \%}$ reduction revealed by the corresponding re-calculated Type-C plans as a reference. The patients are sorted by the PTV size

apatient\# 11 had a minor deviation on $\mathrm{R}_{50 \%}$ even after Type-C re-optimization, while the corresponding original Type-B plan was fully compliant 
Table 7 Plan quality ratios of the re-optimized Type-C plan over the original Type-B plan for the 11 re-optimized patients on PTV coverage $\left(\mathrm{V}_{100 \%}\right)$, Paddick conformity index (PCl) and homogeneity index $(\mathrm{HI})$

\begin{tabular}{lccccc}
\hline Pt \# & $\begin{array}{l}\text { PTV Volume } \\
\left(\mathrm{cm}^{3}\right)\end{array}$ & $\begin{array}{l}\text { D }_{95 \%} \text { Reduction } \\
(\%)\end{array}$ & $\begin{array}{l}\text { Ratio } \\
V_{100 \%}\end{array}$ & $\begin{array}{l}\text { Ratio } \\
\mathrm{PCl}\end{array}$ & $\begin{array}{l}\text { Ratio } \\
\mathrm{HI}\end{array}$ \\
\hline 1 & 6.1 & 12.5 & 1.0 & 1.0 & 1.2 \\
2 & 6.5 & 2.3 & 1.0 & 1.0 & 1.2 \\
3 & 13.0 & 10.6 & 1.0 & 1.0 & 1.1 \\
4 & 13.6 & 15.1 & 1.0 & 1.0 & 1.1 \\
5 & 14.1 & 7.4 & 1.0 & 1.0 & 1.1 \\
6 & 17.0 & 4.1 & 1.0 & 1.0 & 1.2 \\
7 & 18.0 & 4.5 & 1.0 & 1.0 & 1.0 \\
8 & 35.4 & 9.1 & 1.0 & 1.0 & 1.0 \\
9 & 37.1 & 4.7 & 1.0 & 1.1 & 1.0 \\
10 & 48.8 & 9.7 & 1.0 & 1.0 & 1.4 \\
11 & 58.4 & 16.7 & 1.0 & 1.0 & 1.1 \\
\hline
\end{tabular}

Also listed are the PTV volumes and PTV $\mathrm{D}_{95 \%}$ reduction revealed by the corresponding re-calculated Type-C plans

target conformity was compromised, leading to worsened $R_{100 \%}, R_{50 \%}$, and $D_{2 \mathrm{~cm}}$ values. As a result, $R_{100 \%}$ deviation increased from no deviation to minor deviation, $R_{50 \%}$ deviation increased from minor to major deviation, and $\mathrm{D}_{2 \mathrm{~cm}}$ deviation increased from minor to major deviation, which necessitated re-optimization using the Type-C algorithm. On the re-optimized Type- $\mathrm{C}$ plan, the target conformity was restored, and the compliance to the relevant RTOG criteria was also restored to the levels previously achieved by the original Type-B plan.

\section{Discussion}

Our study investigated target dose, RTOG compliance, and other plan quality indexes on 52 lung SBRT patients by comparing between the original Type- $\mathrm{B}$ plan, the recalculated Type-C plan, the re-normalized Type-C plan, and the re-optimized Type-C plan. Although previous studies in the literature have examined dosimetric differences arising from re-calculating Type-B treatment plans with the Type- $\mathrm{C}$ algorithm, discordant recommendations have been made regarding the applicability of current RTOG dosimetric criteria on Type-C calculated lung SBRT plans. Specifically, the study by $\mathrm{Li}$ et al. using Monaco [29] and the studies by Pokhrel et al. using Voxel Monte Carlo [39, 40] suggested that the RTOG dosimetric criteria, such as $R_{100 \%}, R_{50 \%}$, and $D_{2 \mathrm{~cm}}$, might need to be up-adjusted to be more loose. In contrast, the study by Rana et al. using AXB showed comparable or even better compliance for the above criteria [27].

Our study shed some light on the conflicting results above. Similar to Rana et al.'s study [27], the re-calculated Type-C plans also showed comparable or even better compliance to RTOG dosimetric criteria, such as $\mathrm{R}_{100 \%}$, $R_{50 \%}$, and $D_{2 \mathrm{~cm}}$. However, what their study failed to consider was that Type-C re-calculation using the same MLC patterns and MUs would also lead to insufficient target dose coverage and hence sub-optimal plan quality for many patients. In fact, about $13.5 \%$ of our patient cohort showed a PTV $\mathrm{D}_{95 \%}$ reduction over $5 \%$, and about $34.6 \%$ showed a PTV $V_{100 \%}$ reduction over 5\%. When the target coverage loss was made up by re-normalizing the MUs, not surprisingly, compliance to the above RTOG dosimetric criteria worsened for some patients (about 21.2\% of

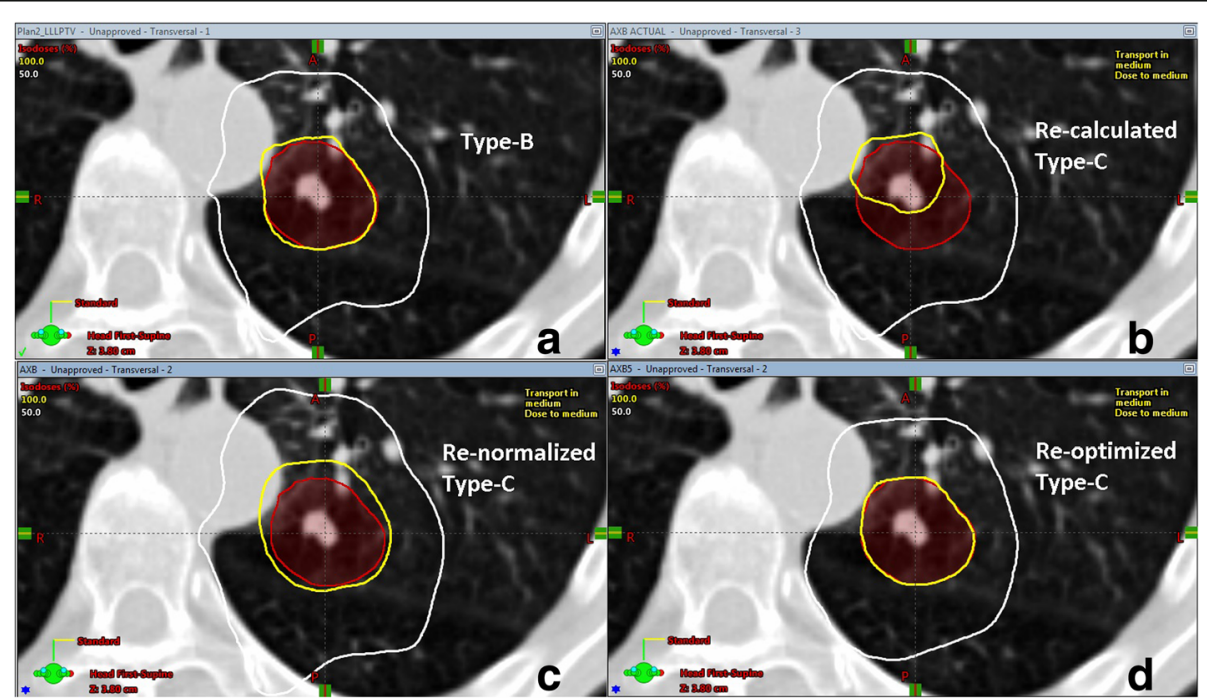

Fig. 4 Axial isodose distributions at the isocenter for an example patient (Patient 3 in Tables 5, 6, and 7) comparing (a) the original Type-B plan, (b) the re-calculated Type-C plan, (c) the re-normalized Type-C plan, and (d) the re-optimized Type-C plan. The PTV is outlined in red colorwash, the $100 \%$ isodose line is marked in yellow, and the $50 \%$ isodose line is marked in white 
our cohort) compared with the original Type-B plans. However, re-optimization using the Type- $\mathrm{C}$ algorithm was able to restore the original RTOG compliance for all but one of these patients. Current RTOG dosimetric criteria hence appear to remain appropriate for lung SBRT plans calculated with Type-C algorithms, provided that such algorithms are employed for plan optimization.

Pokhrel et al. recommended up-adjustments on the values for RTOG dosimetric criteria, such as $R_{100 \%}, R_{50 \%}$, and $\mathrm{D}_{2 \mathrm{~cm}}$, by about $10 \%$ in order for their Type-C plans to comply with the criteria $[39,40]$. However, this was based on the premise that all plans need to fully comply with the criteria. Since in their studies the patients were planned with the Type-C algorithm, and no comparison was made with Type-B plans, it was not considered that for some of these 20 patients, RTOG criteria deviations could have resulted even if planned using Type-B algorithms. In fact, it is not uncommon in current clinical practice for Type-B lung SBRT plans to have minor or even major deviations from these RTOG protocols due to specific patient and tumor anatomy. For example, in the study by Rana et al., among the 14 clinical Type-B plans, 6 plans recorded minor deviations for $R_{100 \%}, 9$ plans recorded minor deviations for $R_{50 \%}$, 4 plans recorded minor deviations for $D_{2 \mathrm{~cm}}$, and 1 plan recorded minor deviation for $\mathrm{V}_{20}$. Similarly, in Li et al.'s series of 15 patients, Type-B plans also resulted in deviations on these RTOG criteria for some of them, such as the 5 minor deviations and 2 major deviations on $\mathrm{D}_{2 \mathrm{~cm}}[29]$.

By designing a comprehensive comparison between original Type- $\mathrm{B}$ plan, the re-calculated Type-C plan, the re-normalized Type-C plan, and the re-optimized Type-C plan, we have clearly outlined in our study the dosimetric necessity as well as impact of re-calculation, re-normalization, and re-optimization using Type-C algorithms. While Li et al.'s study also considered reoptimization using the Type-C algorithm, the conventional conformal beam treatment technique was used in their study, and their re-optimization involved only adjusting the relative beam weights while keeping all other beam parameters the same as the original Type- $B$ plans [29]. Furthermore, we performed our investigation on a much larger patient cohort compared with the above three studies.

Of our 52 patients, $11(21.2 \%)$ resulted in worsened RTOG dosimetric criteria compliance on the re-normalized Type-C plans and hence necessitated re-optimization with the Type- $\mathrm{C}$ algorithm. These patients included all 7 patients with a PTV $\mathrm{D}_{95 \%}$ reduction over $5 \%$. For the remaining 4 patients, 3 had a PTV $D_{95 \%}$ reduction over $4 \%$ and 1 had a reduction of $2 \%$. This indicates that for those patients on whom Type-B algorithms will lead to large PTV coverage dose overestimations, optimization by Type-C algorithms will likely be necessary to achieve similar levels of RTOG dosimetric criteria compliance currently achieved by Type-B algorithms. For patients on whom Type-B algorithms will lead to small PTV coverage dose overestimations, plans optimized with Type-B algorithms will likely only need to be re-normalized with Type-C calculation to ensure sufficient and accurate target dose coverage, as well as to achieve similar levels of RTOG compliance. In a small number of these patients, if the RTOG compliance of the Type-B plans are borderline, optimization with Type- $\mathrm{C}$ algorithms may also be necessary to achieve the same compliance goal.

For one patient, re-optimization using the Type-C algorithm was unable to fully restore the RTOG dosimetric criteria compliance (Patient \#11 in Tables 5, 6, and 7). For this patient, the original plan was fully compliant, and the re-normalized plan resulted in minor deviation in both $R_{50 \%}$ and $D_{2 \mathrm{~cm}}$. The re-optimized plan restored $D_{2 \mathrm{~cm}}$ compliance to no deviation, but on $\mathrm{R}_{50 \%}$ the re-optimized Type-C plan still had a minor deviation. Among our cohort, this patient was also the one with the highest magnitude of PTV $D_{95 \%}$ reduction on the re-calculated Type$\mathrm{C}$ plan, with a reduction of $16.7 \%$. This large discrepancy in target dose calculation between Type-C and Type- $\mathrm{B}$ algorithms might have partially contributed to the difficulty of re-optimizing a fully-compliant plan with the Type- $C$ algorithm. In addition, the $R_{50 \%}$ value of the original Type-B plan for this patient, 3.4, was somewhat close to the minor deviation threshold of 3.8. The re-optimized plan had a value of 4.0, and hence scored as a minor deviation.

For all other patients, re-normalization or reoptimization with the Type-C algorithm achieved similar levels of RTOG compliance for these plans and notably higher target dose heterogeneity as seen in the higher $\mathrm{HI}$ values. The higher $\mathrm{HI}$ might result partially from the fact that the Type-B algorithm overestimates target peripheral dose but underestimates maximum target dose relative to the Type- $\mathrm{C}$ algorithm, and partially from the fact that target dose homogeneity was not heavily constrained during re-optimization. Since the current RTOG protocols recommend a wide range of prescription isodose levels from 60 to $90 \%$, the $\mathrm{HI}$ values on the Type-C plans for all patients fell within this range.

Since their introductions into commercial treatment planning systems, many clinical studies have been conducted to compare dose calculation of Type-C algorithms to algorithms of previous generations [22, 23, $25,31,44-47]$. However, their utilization in clinic practice is still very limited [5]. Two factors contributing to the lack of clinical utilization that motivated our study were: (1) the large patient-to-patient variation of error magnitudes for the current algorithm left it unclear if it is 
necessary to clinically switch the algorithm at the cost of increased computational time. (2) the four existing studies on the applicability of current dosimetric guidelines for Type-C algorithms had apparent disagreements in their findings $[27,29,39,40]$. On the first issue, our study using a large patient cohort provided distribution data of target dose errors associated with Type-B calculation for different patients. Clinicians may use these data to assist making decision whether the algorithm switching is necessary. On the second issue, our study resolved the paradox from the existing literature by demonstrating the difference between recalculated and re-normalized Type-C plans. In addition, the results on re-optimized plans shed additional light on the Type-C applicability of current RTOG guidelines, and further provided clinicians with information on different levels of Type-C involvement in treatment planning necessary to satisfy the dosimetric constraints.

One limitation of our work was that, although extensively comparing the original Type- $\mathrm{B}$ and re-calculated, re-normalized and re-optimized Type- $\mathrm{C}$ plans on a large patient cohort and with a rich pool of important dosimetric endpoints, our comparison was conducted using one Type-B algorithm, AAA, and one Type-C algorithm, AXB. In particular, although usually considered as a Type-B algorithm and currently used as a major clinical treatment planning algorithm [19-21, 28, 48-50], AAA has been shown to perform less accurately in heterogeneous environment than some other Type-B algorithms such as CCC $[18,28,30]$. However, due to the limitations of the treatment planning system, namely Pinnacle does not allow importing plans from other treatment planning systems for re-calculation and it also does not have a Type-C algorithm, a comparison to additional Type-B and Type-C algorithms could not be performed on our cohort.

It would be an interesting direction for future studies to assess the clinical impact of switching from Type-B to Type-C algorithms for lung SBRT. For example, on a cohort of patients treated with Type-B plans, the local failure rates can be compared between those patients with large PTV $\mathrm{D}_{95 \%}$ or $\mathrm{V}_{100 \%}$ reductions upon Type-C re-calculation and those patients with small reductions. This way the clinical significance of the dosimetric differences can be determined. However, due to the relatively small percentage of patients with large dose differences between Type- $\mathrm{B}$ and Type- $\mathrm{C}$ calculations (for example, in our cohort, $13.5 \%$ with a PTV $\mathrm{D}_{95 \%}$ reduction over $5 \%$, or $34.6 \%$ with a PTV $\mathrm{V}_{100 \%}$ reduction over $5 \%$ ) and the very high local control rates usually at around $90 \%$, a very large cohort with sufficient longitudinal follow-up would be necessary to power such a study and illustrate the clinical significance of the dosimetric effects.

\section{Conclusions}

Type-B dose calculation considerably overestimates target dose coverage in lung SBRT for some patients, necessitating Type-C re-normalization or re-optimization. Current RTOG dosimetric criteria appear to remain appropriate in the setting of Type-C calculations.

\section{Abbreviations \\ 3D CT: Three-dimensional computed tomography; 4D CT: Four-dimensional computed tomography; AAA: Analytical Anisotropic Algorithm; AXB: Acuros XB; GTV: Gross tumor volume; HI: Homogeneity index; IMRT: Intensity- modulated radiation therapy; ITV: Internal target volume; LINAC: Linear accelerator; MIP: Maximum intensity projection; NRG/RTOG: National Research Group/Radiation Therapy Oncology Group; NSCLC: Non-small cell lung cancer; PCI: Paddick Conformity Index; PTV: Planning target volume; SABR: Stereotactic ablative radiotherapy; SBRT: Stereotactic body radiotherapy; VMAT: Volumetric-modulated arc therapy}

\section{Acknowledgements}

Not applicable.

\section{Funding}

The study was supported by DZ's faculty diversity grant from University of Nebraska Medical Center.

\section{Availability of data and materials}

Not applicable.

\begin{abstract}
Authors' contributions
$\mathrm{CZ}, \mathrm{NB}, \mathrm{RM}, \mathrm{SW}$, and DZ carried out data collection, CZ and DZ conducted data analysis with the help from all other authors. DZ conceived of, designed, and coordinated the study. DZ and CZ helped to draft the manuscript. All authors participated in writing the manuscript. All authors read and approved the final manuscript.
\end{abstract}

\section{Competing interests}

The authors declare that they have no competing interests.

Consent for publication

Not applicable.

Ethics approval and consent to participate

The study was approved by University of Nebraska Medical Center IRB \# 11014-EP

\section{Publisher's Note}

Springer Nature remains neutral with regard to jurisdictional claims in published maps and institutional affiliations.

\section{Author details \\ ${ }^{1}$ School of Biological Sciences, University of Chicago, Chicago, IL, USA. ${ }^{2}$ Department of Radiation Oncology, University of Nebraska Medical Center, 42nd and Emile St, Omaha, NE 68198, USA. ${ }^{3}$ University of Florida Health Proton Therapy Institute, Jacksonville, FL, USA. ${ }^{4}$ Department of Biological Systems Engineering, University of Nebraska Lincoln, Lincoln, NE, USA. ${ }^{5}$ Department of Radiation Oncology, Fujian Medical University Union Hospital, Fuzhou, Fujian, China.}

Received: 18 January 2017 Accepted: 1 May 2017

Published online: 05 May 2017

\section{References}

1. Siegel RL, Miller KD, Jemal A. Cancer statistics, 2016. CA Cancer J Clin. 2016; 66(1):7-30.

2. Uematsu M, Shioda A, Suda A, et al. Computed tomography-guided frameless stereotactic radiotherapy for stage I non-small cell lung cancer: A 5-year experience. Int J Radiat Oncol Biol Phys. 2001;51(3):666-70. 
3. Timmerman R, Papiez L, McGarry R, et al. Extracranial stereotactic radioablation: Results of a phase I study in medically inoperable stage I non-small cell lung cancer. Chest. 2003;124(5):1946-55.

4. Timmerman R, Paulus R, Galvin J, et al. Stereotactic body radiation therapy for inoperable early stage lung cancer. JAMA. 2010;303(11):1070-6.

5. Guckenberger M, Klement RJ, Allgauer $\mathrm{M}$, et al. Local tumor control probability modeling of primary and secondary lung tumors in stereotactic body radiotherapy. Radiother Oncol. 2016;118(3):485-91.

6. Cai J, Malhotra HK, Orton CG. Point/counterpoint. A 3D-conformal technique is better than IMRT or VMAT for lung SBRT. Med Phys. 2014;41(4):040601.

7. Zhang GG, Ku L, Dilling TJ, et al. Volumetric modulated arc planning for lung stereotactic body radiotherapy using conventional and unflattened photon beams: A dosimetric comparison with 3D technique. Radiat Oncol. 2011;6:152. 717X-6-152.

8. Ong C, Verbakel WF, Cuijpers JP, Slotman BJ, Senan S. Dosimetric impact of interplay effect on RapidArc lung stereotactic treatment delivery. Int J Radiat Oncol Biol Phys. 2011;79(1):305-11.

9. Ong CL, Verbakel WF, Dahele M, Cuijpers JP, Slotman BJ, Senan S. Fast arc delivery for stereotactic body radiotherapy of vertebral and lung tumors. Int J Radiat Oncol Biol Phys. 2012;83(1):e137-43.

10. Stambaugh C, Nelms BE, Dilling T, et al. Experimentally studied dynamic dose interplay does not meaningfully affect target dose in VMAT SBRT lung treatments. Med Phys. 2013;40(9):091710.

11. Navarria P, Ascolese AM, Mancosu P, et al. Volumetric modulated arc therapy with flattening filter free (FFF) beams for stereotactic body radiation therapy (SBRT) in patients with medically inoperable early stage non small cell lung cancer (NSCLC). Radiother Oncol. 2013;107(3):414-8.

12. Zhou S, Zhu X, Zhang M, et al. Estimation of internal organ motion-induced variance in radiation dose in non-gated radiotherapy. Phys Med Biol. 2016; 61(23):8157-79.

13. Chetty IJ, Curran B, Cygler JE, et al. Report of the AAPM task group no. 105: Issues associated with clinical implementation of monte carlo-based photon and electron external beam treatment planning. Med Phys. 2007:34(12):4818-53.

14. Herman Tde L, Gabrish H, Herman TS, Vlachaki MT, Ahmad S. Impact of tissue heterogeneity corrections in stereotactic body radiation therapy treatment plans for lung cancer. J Med Phys. 2010;35(3):170-3.

15. Vanderstraeten $B$, Reynaert N, Paelinck L, et al. Accuracy of patient dose calculation for lung IMRT: A comparison of monte carlo, convolution/ superposition, and pencil beam computations. Med Phys. 2006;33(9):3149-58.

16. Schuring D, Hurkmans CW. Developing and evaluating stereotactic lung RT trials: What we should know about the influence of inhomogeneity corrections on dose. Radiat Oncol. 2008;3:21. 717X-3-21.

17. Xiao Y, Papiez L, Paulus R, et al. Dosimetric evaluation of heterogeneity corrections for RTOG 0236: Stereotactic body radiotherapy of inoperable stage I-II non-small-cell lung cancer. Int J Radiat Oncol Biol Phys. 2009;73(4):1235-42.

18. Aarup LR, Nahum AE, Zacharatou C, et al. The effect of different lung densities on the accuracy of various radiotherapy dose calculation methods: Implications for tumour coverage. Radiother Oncol. 2009;91(3):405-14.

19. Ojala JJ, Kapanen MK, Hyodynmaa SJ, Wigren TK, Pitkanen MA. Performance of dose calculation algorithms from three generations in lung SBRT: Comparison with full monte carlo-based dose distributions. J Appl Clin Med Phys. 2014;15(2):4662.

20. Fogliata A, Vanetti E, Albers D, et al. On the dosimetric behaviour of photon dose calculation algorithms in the presence of simple geometric heterogeneities: Comparison with monte carlo calculations. Phys Med Biol. 2007;52(5):1363-85.

21. Hurkmans CW, Cuijpers JP, Lagerwaard FJ, et al. Recommendations for implementing stereotactic radiotherapy in peripheral stage IA non-small cell lung cancer: Report from the quality assurance working party of the randomised phase III ROSEL study. Radiat Oncol. 2009;4:1. 717X-4-1.

22. Miura $\mathrm{H}$, Masai $\mathrm{N}, \mathrm{Oh} \mathrm{RJ}$, et al. Clinical introduction of monte carlo treatment planning for lung stereotactic body radiotherapy. J Appl Clin Med Phys. 2014;15(1):4202.

23. van der Voort van Zyp NC, Hoogeman MS, van de Water S, et al. Clinical introduction of monte carlo treatment planning: A different prescription dose for non-small cell lung cancer according to tumor location and size. Radiother Oncol. 2010;96(1):55-60.

24. Zhuang T, Djemil T, Qi P, et al. Dose calculation differences between monte carlo and pencil beam depend on the tumor locations and volumes for lung stereotactic body radiation therapy. J Appl Clin Med Phys. 2013;14(2):4011.
25. Liu MB, Eclov NC, Trakul N, et al. Clinical impact of dose overestimation by effective path length calculation in stereotactic ablative radiation therapy of lung tumors. Pract Radiat Oncol. 2013;3(4):294-300.

26. Knoos T, Wieslander E, Cozzi L, et al. Comparison of dose calculation algorithms for treatment planning in external photon beam therapy for clinical situations. Phys Med Biol. 2006;51(22):5785-807.

27. Rana S, Rogers K, Pokharel S, Cheng C. Evaluation of acuros XB algorithm based on RTOG 0813 dosimetric criteria for SBRT lung treatment with RapidArc. J Appl Clin Med Phys. 2014;15(1):4474.

28. Hasenbalg F, Neuenschwander H, Mini R, Born EJ. Collapsed cone convolution and analytical anisotropic algorithm dose calculations compared to VMC++ monte carlo simulations in clinical cases. Phys Med Biol. 2007;52(13):3679-91.

29. Li J, Galvin J, Harrison A, Timmerman R, Yu Y, Xiao Y. Dosimetric verification using monte carlo calculations for tissue heterogeneity-corrected conformal treatment plans following RTOG 0813 dosimetric criteria for lung cancer stereotactic body radiotherapy. Int J Radiat Oncol Biol Phys. 2012;84(2):508-13.

30. Han T, Mikell JK, Salehpour M, Mourtada F. Dosimetric comparison of acuros XB deterministic radiation transport method with monte carlo and model-based convolution methods in heterogeneous media. Med Phys. 2011;38(5):2651-64

31. Fragoso M, Wen N, Kumar S, et al. Dosimetric verification and clinical evaluation of a new commercially available monte carlo-based dose algorithm for application in stereotactic body radiation therapy (SBRT) treatment planning. Phys Med Biol. 2010;55(16):4445-64.

32. Sharma SC, Ott JT, Williams JB, Dickow D. Clinical implications of adopting monte carlo treatment planning for CyberKnife. J Appl Clin Med Phys. 2010; 11(1):3142.

33. Fotina I, Winkler P, Kunzler T, Reiterer J, Simmat I, Georg D. Advanced kernel methods vs. monte carlo-based dose calculation for high energy photon beams. Radiother Oncol. 2009;93(3):645-53.

34. Bush K, Gagne IM, Zavgorodni S, Ansbacher W, Beckham W. Dosimetric validation of acuros XB with monte carlo methods for photon dose calculations. Med Phys. 2011;38(4):2208-21.

35. Fogliata A, Nicolini G, Clivio A, Vanetti E, Cozzi L. Critical appraisal of acuros XB and anisotropic analytic algorithm dose calculation in advanced non-small-cell lung cancer treatments. Int J Radiat Oncol Biol Phys. 2012;83(5):1587-95.

36. Tsuruta $Y$, Nakata $M$, Nakamura $M$, et al. Dosimetric comparison of acuros $\mathrm{XB}, \mathrm{AAA}$, and XVMC in stereotactic body radiotherapy for lung cancer. Med Phys. 2014;41(8):081715

37. RTOG. A randomized phase II study comparing 2 stereotactic body radiation therapy (SBRT(schedules for medically inoperable patients with stage I peripheral non-small cell lung cancer. RTOG0915. 2012.

38. RTOG. Seamless phase $1 /$ II study of stereotactic lung radiotherapy (SBRT) for early stage, centrally located, non-small cell lung cancer (NSCLC) in medically inoperable patients. RTOG 0813. 2012

39. Pokhrel D, Badkul R, Jiang H, Kumar P, Wang F. Technical note: Dosimetric evaluation of monte carlo algorithm in iPlan for stereotactic ablative body radiotherapy (SABR) for lung cancer patients using RTOG 0813 parameters. J Appl Clin Med Phys. 2015;16(1):5058.

40. Pokhrel D, Sood S, Badkul R, et al. Assessment of monte carlo algorithm for compliance with RTOG 0915 dosimetric criteria in peripheral lung cancer patients treated with stereotactic body radiotherapy. J Appl Clin Med Phys. 2016:17(3):277-93.

41. Benedict SH, Yenice KM, Followill D, et al. Stereotactic body radiation therapy: The report of AAPM task group 101. Med Phys. 2010;37(8):4078-101.

42. Paddick I. A simple scoring ratio to index the conformity of radiosurgical treatment plans. technical note. J Neurosurg. 2000;93 Suppl 3:219-22.

43. van't Riet A, Mak AC, Moerland MA, Elders LH, van der Zee W. A conformation number to quantify the degree of conformality in brachytherapy and external beam irradiation: Application to the prostate. Int J Radiat Oncol Biol Phys. 1997:37(3):731-6.

44. Huang $B$, Wu L, Lin P, Chen C. Dose calculation of acuros XB and anisotropic analytical algorithm in lung stereotactic body radiotherapy treatment with flattening filter free beams and the potential role of calculation grid size. Radiat Oncol. 2015;10(1):53. -015-0357-0.

45. Zvolanek $\mathrm{K}, \mathrm{Ma} \mathrm{R}$, Zhou $\mathrm{C}$, et al. Still equivalent for dose calculation in the monte carlo era? A comparison of free breathing and average intensity projection CT datasets for lung SBRT using three generations of dose calculation algorithms. Med Phys. 2017; 10.1002/mp.12193. 
46. Zheng $\mathrm{D}$, Zhang $\mathrm{Q}$, Liang $\mathrm{X}$, et al. Effect of the normalized prescription isodose line on the magnitude of monte carlo vs. pencil beam target dose differences for lung stereotactic body radiotherapy. J Appl Clin Med Phys. 2016;17(4):48-58

47. Zheng D, Zhu X, Zhang Q, et al. Target dose conversion modeling from pencil beam (PB) to monte carlo (MC) for lung SBRT. Radiat Oncol. 2016;11:83. 016-0661-3.

48. De Ruysscher D, Faivre-Finn C, Nestle U, et al. European organisation for research and treatment of cancer recommendations for planning and delivery of high-dose, high-precision radiotherapy for lung cancer. J Clin Oncol. 2010;28(36):5301-10.

49. Guckenberger M, Allgauer M, Appold S, et al. Safety and efficacy of stereotactic body radiotherapy for stage 1 non-small-cell lung cancer in routine clinical practice: A patterns-of-care and outcome analysis. J Thorac Oncol. 2013;8(8):1050-8.

50. van Baardwijk A, Tome WA, van Elmpt W, et al. Is high-dose stereotactic body radiotherapy (SBRT) for stage I non-small cell lung cancer (NSCLC) overkill? A systematic review. Radiother Oncol. 2012;105(2):145-9.

Submit your next manuscript to BioMed Central and we will help you at every step:

- We accept pre-submission inquiries

- Our selector tool helps you to find the most relevant journal

- We provide round the clock customer support

- Convenient online submission

- Thorough peer review

- Inclusion in PubMed and all major indexing services

- Maximum visibility for your research

Submit your manuscript at www.biomedcentral.com/submit
Biomed Central 